\title{
Results Towards Identifiability Properties of Biochemical Reaction Networks
}

\author{
Marcello Farina, Rolf Findeisen, Eric Bullinger, \\ Sergio Bittanti, Frank Allgöwer, Peter Wellstead
}

\begin{abstract}
In this paper we consider the question of parameter identifiability for biochemical reaction networks, as typically encountered in systems biology. Specifically, we are interested in deriving conditions on the biochemical reaction network and on the measured outputs that guarantee identifiability of the parameters. Taking the specific system structure of biochemical reaction networks into account, we derive sufficient conditions for local parameter identifiability based on a suitable system expansion which does not anymore directly depend on the parameters. Rather, as shown, the problem of identifiability can be recast as the question of observability of the (parameter free) expanded system. The conditions derived are exemplified considering a simple example.
\end{abstract}

Index Terms-Chemical reaction networks, identifiability, parameter identification, systems biology.

\section{INTRODUCTION}

The last decades have seen rapid advancements in the understanding of biological behaviour on a sub-cellular and cellular level. These advancements are part-wise driven by developments in proteomics, genomics, and measurement technologies. This rapid advancement has lead to a significant increase in knowledge, data, and information available on the sub-cellular and cellular level. However, by now it has become clear that the identification of single components, such as genes or proteins does not lead to a complete picture and understanding of the biological processes involved. Instead, a holistic understanding requires a systems approach including mathematical modelling and analysis. This awareness has lead to the research direction of systems biology [12], [24].

One of the central themes in systems biology is the mathematical modelling, dynamic simulation, and analysis of metabolic and signal transduction pathways. Typically, the modelling based on biochemical reaction networks leads to a high number of states and differential equations, with a large number of parameters describing the reaction kinetics. Examples are models of signalling cascades which have up to several hundred states and even more parameters, see for

Marcello Farina and Sergio Bittanti are with the Dipartimento di Elettronica e Informazione, Politecnico di Milano, Milan, Italy \{farina, bittanti\}@elet.polimi.it

Rolf Findeisen, Eric Bullinger, and Frank Allgöwer are with the Institute for Systems Theory and Automatic Control, University of Stuttgart, Germany \{findeise, allgower\} @ist.uni-stuttgart.de

Eric Bullinger and Peter Wellstead are with the Hamilton Institute, National University of Ireland, Maynooth, Ireland \{eric.bullinger, peter.wellstead\} @nuim.ie

* mf, rf and eb contributed equally to this work.

Major parts of this work have been done while Marcello Farina has been at the Institute for Systems Theory and Automatic Control on a Marie Curie Fellowship of the European Community in the framework of the European Control Training Site (CTS), contract number HPMT-CT-2001-00278. example [21] for a specific example with about 120 states, or [27] for an overview.

Often, the parameters are either completely unknown, or only rough estimates for them are available. Since the behaviour and dynamics of the network strongly depend on these parameters, estimating them from experimental data is a significant bottleneck in systems biology. Theoretically as well as practically, there are many open questions concerning the parameter identification for biochemical networks, spanning from the rather large measurement errors typically encountered in systems biology, the typical lack of reliable dynamical measurement data, up to the question of parameter identification of continuous time systems.

We consider the problem of parameter identification from a systems perspective. Taking the specific system structure of biochemical reaction networks into account, we derive sufficient conditions for local parameter identifiability based on a suitable system expansion. The presented results should be seen as preliminary results, laying a theoretically sound basis for the development of new identification methodologies for biochemical reaction networks.

The paper is structured as follows. In Section II we outline the problem of parameter identification for chemical reaction networks and review the current state of the art. In Section III we state the main result, giving sufficient conditions for local parameter identifiability for chemical reaction networks. As shown, the results are based on a suitable system expansion and considering the problem of identifiability as the question of local observability of the expanded system. Section IV shows the application of the results to the question of parameter identifiability in the case of a simple example system, before we conclude in Section V.

\section{Parameter Identification for Chemical REACTION NETWORKS: STATE OF THE ART}

We are interested in the question of parameter identification and identifiability for biochemical reaction networks. Typically, biochemical reaction networks are described in terms of the $n$ dimensional state vector $c \in \mathbb{R}^{n}$ of concentrations of the species taking part in the reactions:

$$
\dot{c}=N v(c, \theta) .
$$

Here $N \in \mathbb{R}^{n \times r}$ is the so called stoichiometric matrix, where $r$ is the number of reactions taking place. For each reaction, $v: \mathbb{R}^{n} \times \mathbb{R}^{p} \rightarrow \mathbb{R}^{r}$ describes the reaction rate. Here $\theta \in \mathbb{R}^{p}$ are the (unknown) reaction parameters. Typically, the reaction rate is a nonlinear function of the concentrations and of the parameters. Classical examples are the well known 
Michaelis-Menten kinetics, the Hill kinetics, and simple expressions in terms of the so-called mass action law, see e.g. [5] for an introduction. In the following we will focus on kinetics described by the mass action law. One of the reasons for this is that many kinetics, such as the MichaelisMenten kinetics, can be derived from the mass action law by limit considerations. Additionally, as will become clear, considering the special structure of kinetics described by the mass action law allows us to reformulate the system in a way which makes it especially suitable for identification purposes. Especially it allows considering the identification problem as an observation problem of an expanded state vector.

Kinetics following the law of mass action actually assume that each reaction rate $v_{k}$ appearing in the chemical pathway is given by a term proportional to the concentrations of the reacting species and a rate constant $\theta_{k}$, i.e.:

$$
v_{k}=\theta_{k} \prod_{i=1}^{n} c_{i}^{\eta_{k i}}
$$

Here the constants $\eta_{k i}$ are integers representing the stoichiometric amount of the species $i$ taking part in the $k$-th reacting complex. For instance, $\eta_{k i}=0$, if species $i$ is not a substrate of reaction $k$. For an overview on mass action reaction networks and their properties see for example [10], [4].

Remark 1: In Section III we need a special "factorisation" of the stoichiometric matrix $\mathrm{N}$ similar to the one used in [9], which is, as will become clear, particularly useful to stress the linear dependency of the system on the parameters. Specifically, the entries in $N$ are split in positive and negative entries, i.e. entries which are positive are placed into the output stoichiometric matrix $N_{\text {out }} \in \mathbb{R}^{n \times r}$, whereas negative entries are collected in the input stoichiometric matrix $N_{\text {in }} \in$ $\mathbb{R}^{n \times r}$ such that

$$
N=N_{\text {out }}-N_{\text {in }} \text {. }
$$

Note that for mass action law kinetics the input stoichiometric matrix is given by

$$
N_{\mathrm{in}}^{i k}=\eta_{k i}
$$

where $\eta_{k i}$ corresponds to those of (2). A non-zero entry at $(i, j)$ in $N_{\text {in }}$ thus corresponds to the fact that species $i$ is consumed by reaction $j$.

The main objective of this paper is to derive conditions under which the parameters $\theta$ of (1) can be identified from measurements as typically available in systems biology. In the paper we assume that the outputs used for the parameter estimation are of the form:

$$
y=h(c, v) .
$$

Typically, linear functions of the concentrations and the reaction rates as outputs are consistent with biological experiments. This is also good for theoretical considerations, since it simplifies the identification problem, avoiding, for example, output multiplicities. Thus we restrict the class of outputs to linear combinations of the concentrations or of the reaction rates:

$$
y=\left[\begin{array}{l}
y_{c} \\
y_{v}
\end{array}\right]=\left[\begin{array}{cc}
H_{c} & 0 \\
0 & H_{v}
\end{array}\right]\left[\begin{array}{l}
c \\
v
\end{array}\right]
$$

Remark 2: In general, the matrices $H_{c}$ and $H_{v}$ can have an arbitrary structure. However, in experiments often single concentration, or reaction rates are measured. Thus, the rows of the matrices $H_{v}$ and $H_{c}$ are normally vectors having only one non-zero element.

As outlined in the following, identifying the parameter from the available data is a challenging problem, theoretically as well as practically.

\section{A. Challenges and review of existing approaches for param- eter identification of chemical reaction networks}

In biological system modelling, parameter estimation is commonly achieved using global search algorithms, see e.g. [15], [7].

Beside the high demand on computation power, global approaches give usually neither a guarantee of convergence nor a measure of how close their solution is to the optimal approximation. Partial solutions are local identifiability analyses [28] or algebraic approaches [1], which only work in very low state dimensions.

There is therefor a need of developing identification algorithms that take into account the specificities of biochemical reaction network models to provide estimates with error bounds and that can be applied to the typical size of several hundred concentration and reaction rates.

The problem of parameter identification in systems describing the behaviour of chemical reactions networks is a very peculiar and challenging problem. The problem of identification of nonlinear continuous time systems is, in fact, an issue which has not commonly been explored (see, for example, [26] and [22]), as well as for nonlinear systems with fixed non canonical structure, see [17], [11], [18], [19]. For example, when dealing with systems that are not in an observer normal form, or which are not in some kind of an external representation, there exists no general solution to the problem by now. In addition, the data available are generally sampled, and there are (experimental) big constraints on which specific variables can be measured. Last, one condition we generally need to satisfy in order to achieve the property of practical identifiability is the possibility to excite the system with a given input signal. In the biological framework, the possibility offered of exciting the system with sufficiently rich inputs is typically very limited.

Several efforts have addressed the posed problem. The most common approach used in literature makes use of the Prediction Error Minimisation method (PEM). This approach has been adopted both in more practical-oriented works (see [3], [25]) and in a more theoretical framework. In fact, the problem of experimental design have been deeply treated (see, for example [13], [2], [6], and others) and several conditions based on optimisation related to the Fisher matrix (arising from the Cramer-Rao inequality theory (see [14])) 
have been proposed. Solutions of the experimental design problem are normally given through numerical calculation, and are not closely related to the structure of the system.

Furthermore, the solution of the global optimisation problem arising from posing the PEM problem is also given by numerical optimisation algorithms. Several different optimisation methods have been compared [16], obtaining the results that global gradient-based methods, deterministic and stochastic strategies can in general not guarantee optimality of their solution. Furthermore such methods do not give any condition to assess existence of a unique minimum of the cost function.

An approach based on assumptions of optimality of biological systems has been recently proposed [8], which provides a powerful and biologically consistent method. From a mathematical point of view, this approach, however, does not guarantee the solution to be equal to the "ideal" parametrisation of the real system model, and it does not provide structural conditions assessing identifiability.

In the frame of this paper, we set out to provide at least partial answers to the appearing problems. Especially, we focus on the problem of deriving identifiability conditions for biochemical reaction networks.

\section{LOCAL IDENTIFIABILITY OF CHEMICAL REACTION NETWORKS}

In this section we derive conditions that guarantee local identifiability of biochemical reaction networks based on a time-varying linearisation along system trajectories. The idea is based on a suitable system expansion, allowing to reformulate the problem in terms of the fluxes and concentrations solely. The results obtained should be seen as preliminary in nature.

We assume for simplicity that

Assumption 1: Concentrations and parameters are strictly positive.

Remark 3: This assumption is common in modelling and analysing biochemical reaction networks. We do not go into details under which conditions positivity of (1) can be guaranteed starting in the positive state space.

Assuming that Assumption 1 holds, we can derive for the class of biochemical reaction networks modelled by mass action laws as described in Section II the following result.

Theorem 1: Under Assumption 1 the system (1) is equivalent to the expanded system

$$
\frac{d}{d t}\left[\begin{array}{l}
c \\
v
\end{array}\right]=\left[\begin{array}{c}
f_{c}(v) \\
f_{v}(c, v)
\end{array}\right]
$$

with

$$
\begin{aligned}
f_{c}(v) & =N v \\
f_{v}(c, v) & =\operatorname{diag}(v) N_{\text {in }}^{T}(\operatorname{diag}(c))^{-1} N v .
\end{aligned}
$$

Furthermore, identification of the parameters $\theta$ of (1) based on the outputs (5) is equivalent to observability of (6).
Proof: (of Theorem 1)

We first expand (1) with a trivial differential equation for the parameters

$$
\begin{aligned}
& \dot{c}=N v(c ; \theta) \\
& \dot{\theta}=0 .
\end{aligned}
$$

Combining the mass action law with the definition of the input stoichiometry leads to:

$$
v=\operatorname{diag}(\theta) \exp \left(N_{\text {in }}^{T} \log (c)\right),
$$

where $\log (\cdot)$ and $\exp (\cdot)$ are element-wise operators. Applying the logarithm operator to (8) yields

$$
\log (v)=\log (\theta)+N_{\text {in }}^{T} \log (c) .
$$

Finally, taking the derivative with respect to time leads to

$$
(\operatorname{diag}(v))^{-1} \dot{v}=N_{\text {in }}^{T}(\operatorname{diag}(c))^{-1} \dot{c},
$$

as the parameters $\theta$ are constant. This allows to transform (7) into the equivalent system description (6).

Remark 4: The expanded system (6) has $n+r$ states and is formulated in terms of the concentrations and flows. Furthermore, the parameters $\theta$ do not appear explicitly anymore. They are rather "hidden" in the initial condition of the reaction rate $v$. One advantage of the description (6) is that usually biological experiments measure certain concentration, the sum of different species concentration and/or certain reaction rates. Then, the output is simply a linear mapping of the states. Classically, the measurement of reaction rates leads to output nonlinearities. Here, the corresponding outputs are also linear functions of the states.

The main advantage of using this approach is that the problem of identification can be cast as a pure observation problem, i.e. the question of identifiability is transfered to the question of observability of (6). Thus, a suitable observer for the expanded system would estimate both the initial species concentration $c$ and thus the parameters $\theta$.

This observation is captured by the following result:

Theorem 2: If along a trajectory $(\bar{c}, \bar{v})=(\bar{c}(t), \bar{v}(t))$, the linearisation of (6) with the output (5) is observable, then the parameters $\theta$ of (1) can be locally identified from the output measurements (5).

\section{Proof: (of Theorem 2)}

According to Theorem 1, the question of parameter identifiability of (1) via the output (5) is equivalent to the observability of (6) via the output (5). Following [23], [20] local observability of (6) is equivalent to observability of the (time varying) around a trajectory linearised system.

The following result states the importance of excitation of the system.

Corollary 1: A necessary condition for observability is that $H_{c}$ has full rank.

Proof: The linearisation of the system (6) around a steady-state $(\bar{c}, \bar{v})$ is given, using

$$
\left[\begin{array}{l}
c \\
v
\end{array}\right]=\left[\begin{array}{l}
\bar{c} \\
\bar{v}
\end{array}\right]+\left[\begin{array}{l}
\Delta c \\
\Delta v
\end{array}\right],
$$


by

$$
\left[\begin{array}{c}
f_{c}(\Delta v) \\
f_{v}(\Delta c, \Delta v)
\end{array}\right]=J(\bar{c}, \bar{v})\left[\begin{array}{c}
\Delta c \\
\Delta v
\end{array}\right]+\left[\begin{array}{c}
0 \\
\mathcal{O}(\bar{c}, \bar{v})^{2}
\end{array}\right]
$$

where the Jacobian matrix $J$ of the linearised extended system is given by

$$
J(\bar{c}, \bar{v})=\left[\begin{array}{cc}
0 & N \\
J_{21}(\bar{c}, \bar{v}) & J_{22}(\bar{c}, \bar{v})
\end{array}\right]
$$

with

$$
\begin{aligned}
J_{21}(\bar{c}, \bar{v})= & \frac{\partial}{\partial c}\left(\operatorname{diag}(v) N_{\text {in }}^{T}\right. \\
& \left.\cdot \operatorname{diag}(N v)(\operatorname{diag}(c))^{-1}\left[\begin{array}{c}
1 \\
\ldots \\
1
\end{array}\right]\right)\left.\right|_{(c, v)=(\bar{c}, \bar{v})} \\
= & \left.\operatorname{diag}(v) N_{\text {in }}^{T} \operatorname{diag}(N v)(\operatorname{diag}(c))^{-2}\right|_{(c, v)=(\bar{c}, \bar{v})} \\
= & \operatorname{diag}(\bar{v}) N_{\text {in }}^{T} \operatorname{diag}(N \bar{v})(\operatorname{diag}(\bar{c}))^{-2} \\
= & 0
\end{aligned}
$$

and

$$
\begin{aligned}
J_{22}(\bar{c}, \bar{v})= & \left.\frac{\partial}{\partial v}\left(\operatorname{diag}(v) N_{\text {in }}^{T}(\operatorname{diag}(c))^{-1} N v\right)\right|_{(c, v)=(\bar{c}, \bar{v})} \\
= & \left.\operatorname{diag}(v) N_{\text {in }}^{T}(\operatorname{diag}(c))^{-1} N\right|_{(c, v)=(\bar{c}, \bar{v})} \\
& \quad+\left.\operatorname{diag}\left(N_{\text {in }}^{T}(\operatorname{diag}(c))^{-1} N v\right)\right|_{(c, v)=(\bar{c}, \bar{v})} \\
= & \operatorname{diag}(\bar{v}) N_{\text {in }}^{T}(\operatorname{diag}(\bar{c}))^{-1} N \\
= & \bar{J}_{22} .
\end{aligned}
$$

The linearisation of (6) around $(\bar{c}, \bar{v})$ is therefore given by

$$
\begin{aligned}
& \dot{x}=J(\bar{c}, \bar{v})\left[\begin{array}{l}
\Delta c \\
\Delta v
\end{array}\right]=\left[\begin{array}{ll}
0 & N \\
0 & \bar{J}_{22}
\end{array}\right]\left[\begin{array}{l}
\Delta c \\
\Delta v
\end{array}\right], \\
& y=H\left[\begin{array}{l}
\Delta c \\
\Delta v
\end{array}\right] .
\end{aligned}
$$

Clearly, a necessary condition for observability is that $H_{c}$ has full rank.

The last result states the importance of knowledge of dynamical data (and of excitation of the system) for the observation (and thus for identification) purposes.

\section{EXAMPLE}

In order to clarify the results obtained and the extended system structure, we consider a simple example, for which we will especially verify the derived identifiability conditions.

We consider a very simple system of two concentrations and two reaction rates of the form:

$$
\begin{gathered}
v_{1}: A \rightarrow B \\
v_{2}: B \rightarrow A .
\end{gathered}
$$

This system can be described by

$$
\frac{d}{d t}\left[\begin{array}{l}
c_{1} \\
c_{2}
\end{array}\right]=N\left[\begin{array}{l}
v_{1} \\
v_{2}
\end{array}\right]
$$

where

$$
\begin{aligned}
& N=\left[\begin{array}{cc}
-1 & 1 \\
1 & -1
\end{array}\right], \\
& v_{1}=\theta_{1} c_{1} \\
& v_{2}=\theta_{2} c_{2} .
\end{aligned}
$$

We first derive the expanded system description (13). For this, note that

$$
N_{\text {in }}=\left[\begin{array}{ll}
1 & 0 \\
0 & 1
\end{array}\right], \quad N_{\text {out }}=\left[\begin{array}{ll}
0 & 1 \\
1 & 0
\end{array}\right],
$$

and

$$
N=N_{\text {out }}-N_{\text {in }}
$$

The new description of the system is given by

$$
\frac{d}{d t}\left[\begin{array}{l}
c \\
v
\end{array}\right]=\left[\begin{array}{c}
N v \\
\operatorname{diag}(v) N_{\text {in }}^{T}(\operatorname{diag}(c))^{-1} N v
\end{array}\right] .
$$

Using $N_{i n}$ as defined above, this simplifies to

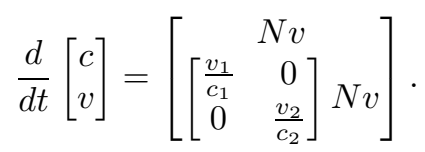

At an arbitrary state $(\bar{c}, \bar{v})$, the Jacobi-matrix is given by

$$
J(\bar{c}, \bar{v})=\left[\begin{array}{cc}
0 & N \\
J_{21}(\bar{c}, \bar{v}) & J_{22}(\bar{c}, \bar{v})
\end{array}\right]
$$

where

$$
\begin{aligned}
& J_{21}(\bar{c}, \bar{v})=\left(\bar{v}_{2}-\bar{v}_{1}\right)\left[\begin{array}{cc}
-\frac{\bar{v}_{1}}{\bar{c}_{1}^{2}} & 0 \\
0 & \frac{\bar{v}_{2}}{\bar{c}_{2}^{2}}
\end{array}\right], \\
& J_{22}(\bar{c}, \bar{v})=\left[\begin{array}{cc}
\bar{v}_{1} & 0 \\
\bar{c}_{1} & \bar{v}_{2} \\
0 & \bar{c}_{2}
\end{array}\right] N+\left[\begin{array}{cc}
\frac{\bar{v}_{2}-\bar{v}_{1}}{\bar{c}_{1}} & 0 \\
0 & \frac{\bar{v}_{1}-\bar{v}_{2}}{\bar{c}_{2}}
\end{array}\right] .
\end{aligned}
$$

By Assumption 1, $J_{21}$ has full rank if $\bar{v}_{1} \neq \bar{v}_{2}$. This is satisfied whenever the system is not in steady-state.

We first check the Hautus test in steady-state, where $\bar{v}_{1}=$ $\bar{v}_{2}$. Then

$$
J_{21}=0
$$

and

$$
J_{22}=\left[\begin{array}{cc}
-\frac{\bar{v}_{1}}{\bar{c}_{1}} & 0 \\
0 & -\frac{\bar{v}_{2}}{\bar{c}_{2}}
\end{array}\right] N
$$

and the observability is equivalent to full rank of the following matrix

$$
\left[\begin{array}{cc}
\lambda I & -N \\
0 & \lambda I-J_{22} \\
H_{c} & H_{v}
\end{array}\right] \forall \lambda \in \mathbb{C} .
$$


From $\lambda=0$ follows that full rank of $H_{c}$ is necessary for full rank of (18). Note that $[1,1]^{T}$ is in the kernel of $N$, and thus $[0,0,1,1]^{T}$ is $\mathrm{n}$ the kernel of $J$. Thus, is in steady-state, the matrix (18) has full rank for $\lambda=0$ only if $[1,1]^{T}$ is not in the kernel of $H_{v}$.

The non-zero Eigenvalues of $J$ are the non-zero Eigenvalues of $J_{22}$. For the given stoichiometry $N$, we can calculate these analytically:

$$
J_{22}=\left[\begin{array}{cc}
\theta_{1} & 0 \\
0 & \theta_{2}
\end{array}\right]\left[\begin{array}{cc}
-1 & 1 \\
1 & -1
\end{array}\right]=\left[\begin{array}{cc}
-\theta_{1} & \theta_{1} \\
\theta_{2} & -\theta_{2}
\end{array}\right] .
$$

Thus, $J_{22}$ has as Eigenvalues $\lambda_{1}=0$ and $\lambda_{2}=-\theta_{1}-\theta_{2}$. The Eigenvector to $\lambda_{2}, v_{2}$ is

$$
v_{2}=\left[\begin{array}{l}
\theta_{1} \\
\theta_{2}
\end{array}\right] .
$$

Thus, the steady-state $(\bar{c}, \bar{v})$ is observable if and only if $H_{c}$ has full rank and $v_{2}$ is not in the kernel of $H_{v}$.

We now analyse observability along a (non-constant) trajectory $(\bar{c}, \bar{v})$ for $H_{v}=0$ and $H=I$. The observability map of the nonlinear system can be explicitly calculated. The output together with the output derivatives $\dot{y}_{1}$ and $\ddot{y}_{1}$ results in

$$
\left[\begin{array}{l}
y_{1} \\
y_{2} \\
\dot{y}_{1} \\
\ddot{y_{1}}
\end{array}\right]=\left[\begin{array}{c}
\bar{c}_{1} \\
\bar{c}_{2} \\
\left(\bar{v}_{2}-\bar{v}_{1}\right) \\
-\left(\bar{v}_{2}-\bar{v}_{1}\right)\left(\frac{\bar{v}_{2}}{\bar{c}_{2}}+\frac{\bar{v}_{1}}{\bar{c}_{1}}\right)
\end{array}\right]
$$

which can be analytically inverted in a straight-forward way whenever $v_{1} \neq v_{2}$, i.e. for non-steady-state $(\bar{c}, \bar{v})$. Thus, the system is locally uniformly observable. As a third derivative of $y_{1}$ is a function of $\dot{y}_{1}$ and $\ddot{y}_{1}$ and $y_{2}^{(i)}=-y_{1}^{(i)}$, clearly both concentrations need to be measured for observability to hold. Since eq. (19) can be analytically inverted, the system results to be globally uniformly observable. As mentioned, since state variables $c$ and $v$ can be recovered, through a least square procedure the values of parameters $\theta$ can be estimated.

A Hautus-test of this example along a trajectory is a nice illustration of the necessity of calculation it for a timevarying system, see e.g. [20]: While the matrix

$$
\left[\begin{array}{c}
H \\
H J \\
H J^{2} \\
H J^{3}
\end{array}\right]
$$

has full rank,

$$
\left[\begin{array}{c}
H \\
H J \\
H J^{2}+H \dot{J} \\
H J^{3}+H \dot{J} J+H J \dot{J}
\end{array}\right]=\left[\begin{array}{c}
H \\
H J \\
H J^{2} \\
H J^{3}+H J \dot{J}
\end{array}\right]
$$

has not, consistently with the result of the nonlinear observability map.

\section{CONCLUSIONS}

In the present work the problem of developing criteria to assess parametric identifiability of biochemical reaction network has been explored.

This problem, as well as the problem of developing theoretically sound algorithms for identification, is crucial in systems biology, since the parameters appearing in the models are in general difficult to obtain and are only approximately known. However, the qualitative behaviour of the system can depend significantly on these parameters, and thus obtaining good estimates of them is of crucial interest for future developments.

The present work states new conditions to guarantee identifiability of models. It might provide paths for the exploration of new methodologies and might suggests new directions, which can lead to practical solutions of the identification problem.

\section{ACKNOWLEDGEMENTS}

The work was supported through a European Community Marie Curie Fellowship in the frame of the European Control Training Site (CTS), contract number: HPMT-CT2001-00278. EB and PW acknowledge funding from Science Foundation Ireland (SFI) grant 03/RP1/I382.

\section{REFERENCES}

[1] S. Audoly, G. Bellu, L. D'Angiò, M. P. Saccomani, and C. Cobelli. Global identifiability of linear models of biological systems. IEEE Transactions on Biomedical Engineering, 48(1):55-65, 2001.

[2] M. Baltes, R. Schneider, C. Sturm, and M. Reuss. Optimal experimental design for parameter estimation in unstructured growth models. Biotechnol. Prog., 10:480-488, 1994.

[3] M. Bentele, I. Lavrik, M. Ulrich, S. Stößer, D. Heermann, H. Kalthoff, P. Krammer, and R. Eils. Mathematical modeling reveals threshold mechanism in CD95-induced apoptosis. Journal of Cell Biology, 166(6):839-851, 2004.

[4] M. Chaves and E. D. Sontag. State-estimation for chemical reaction networks of Feinberg-Horn-Jackson zero deficiency type. European Journal of Control, 8:343-359, 2002.

[5] C. P. Fall, E. S. Marland, J. M. Wagner, and J. J. Tyson, editors. Computational Cell Biology. Springer, 2002.

[6] D. Faller and D. Klingmueller. Simulation methods for optimal experimental design in systems biology. Simulation, 79(12):717-725, 2003.

[7] X.-J. Feng and H. Rabitz. Optimal identification of biochemical reaction networks. Biophysical Journal, 86(3):1270-1281, 2004.

[8] K. G. Gadkar, R. Gunawan, and F. J. Doyle III. Iterative approach to model identification of biological systems. BMC Bioinformatics, 6(155), 2005.

[9] K. Gatermann and B. Huber. A family of sparse polynomial systems arising in chemical reaction systems. Journal of Symbolic Computation, 33:275-305, 2002.

[10] J. M. Gubermann. Mass action reaction networks and the zero deficiency theorem. Master's thesis, Harvard University, Cambridge, 2003.

[11] V. R. Karanam, P. A. Frick, and R. R. Mohler. Bilinear system identification by Walsh functions. IEEE Transaction on Automatica Control, 23(4):709-713, 1978.

[12] H. Kitano. Systems biology: A brief overview. Science, 295:16621664, 2002.

[13] Z. Kutalik, K.-H. Cho, and O. Wolkenhauer. Optimal sampling time selection for parameter estimation in dynamic pathway modeling. Biosystems, 75:43-55, 2004.

[14] L. Ljung. System Identification - Theory for the User. Prentice Hall, 2nd edition, 1999. 
[15] C. G. Moles, P. Mendes, and J. R. Banga. Parameter estimation in biochemical pathways: a comparison of global optimization methods. Genome Res, 13(11):2467-2474, Nov. 2003.

[16] C. G. Moles, P. Mendes, and J. R. Banga. Parameter estimation in biochemical pathways: A comparison of global optimization methods. Genome Research, 13:2467-2474, 2003.

[17] O. Nelles. Nonlinear Systems Identification. Springer, 2001.

[18] M. Niethammer. An approach towards continuous-time nonlinear system identification. Master's thesis, Institute for Systems Theory in Engineering, University of Stuttgart, Stuttgart, 2000.

[19] M. Niethammer, P. H. Menold, and F. Allgöwer. Parameter and derivative estimation for nonlinear continuous-time system identification. Proceedings of the 5th IFAC Symposium on Nonlinear Systems, NOLCOS 01, pages 691-696, 2001.

[20] W. J. Rugh. Linear Systems Theory. Prentice Hall, Englewood Cliffs, NJ, 1996.

[21] B. Schoeberl, C. Eichler-Jonsson, E. D. Gilles, and G. Müller. Computational modeling of the dynamics of the MAP kinase cascade activated by surface and internalized EGF receptors. Nat. Biotechnol., 20(4):370-375, 2002.

[22] N. K. Sinha and G. P. Rao. Identification of continuous-time systems, methodology and computer implementation. Kluwer Academic Publishers, 1991.

[23] E. Sontag. Mathematical Control Theory. Springer-Verlag, New York, 1998.

[24] E. D. Sontag. Molecular systems biology and control. European Journal of Control, 11:396-435, 2005.

[25] J. Stelling and E. D. Gilles. Robustness vs. identifiability of regulatory modules? The case of mitotic control in budding yeast cell cycle regulation. Proc. 2nd International Conference on Systems Biology, pages 181-190, 2001.

[26] H. Unbehauen and G. P. Rao. A review of identification in continuoustime systems. Annual Reviews in Control, 22:145-171, 1998.

[27] S. J. Vayttaden, S. M. Ajay, and U. S. Bhalla. A spectrum of models of signaling pathways. ChemBioChem, 5(10):1365-1374, Oct 2004.

[28] D. E. Zak, G. E. Gonye, J. S. Schwaber, and F. J. Doyle III. Importance of input perturbations and stochastic gene expression in the reverse engineering of genetic regulatory networks: Insights from an identifiability analysis of an in silico network. Genome Research, 13(11):2396-2405, 2003. 